\title{
Generalised Weighted Relevance Aggregation Operators for Hierarchical Fuzzy Signatures
}

\author{
B. S. U. Mendis ${ }^{I}$ \\ ${ }^{I}$ Dept. of Computer \\ Science \\ The Australian \\ National University \\ Canberra, Australia \\ sumudu@cs.anu.edu.au
}

\author{
T. D. Gedeon \\ College of Eng. and \\ Computer Science \\ The Australian \\ National University \\ Canberra, Australia \\ tom@cs.anu.edu.au
}

\author{
J. Botzheim ${ }^{1,2}$ \\ ${ }^{2}$ Dept. of Telec. \& \\ Media Informatics, \\ Budapest University \\ of Tech. \& Econs, \\ Budapest, Hungary. \\ botzheim@tmit.bme.hu
}

\author{
L. T. Kóczy ${ }^{2,3}$ \\ ${ }^{3}$ Institute of Info. Tech. \\ and Elec. Eng., \\ Széchenyi István \\ University, Györ, \\ Hungary \\ koczy@tmit.bme.hu
}

\begin{abstract}
Hierarchical Fuzzy Signatures are generalizations of the Vector Valued Fuzzy Set concept introduced in the 1970s. A crucial question in the Fuzzy Signature context is what kinds of aggregations are applicable for combining data with partly different substructures. Our earlier work introduced the Weighted Relevance Aggregation method to enhance the accuracy of the final results of calculations based on Hierarchical Fuzzy Signature Structures. In this paper, we further generalise the weights and the aggregation into a new operator called Weighted Relevance Aggregation Operator (WRAO). WRAO enhances the adaptability of the fuzzy signature model to different applications and simplifies the learning of fuzzy signature models from data. We also show the methodology of learning these aggregation operators from data.
\end{abstract}

\section{Introduction}

In Kóczy [8] the vector valued fuzzy sets concept [7] has been further generalised to introduce the fuzzy signature concept. Fuzzy signatures can model complex structured problems via hierarchically structured (embedded) vector valued fuzzy sets, and a set of aggregation functions. The hierarchically structured vector valued fuzzy sets represent the degree of relationship of the attributes of the object. The set of aggregation functions tunnel, from lower branches to the higher branches, the interconnectedness of different universes of discourses of hierarchical fuzzy signature structure. Also, these aggregation functions are non- homogenous, that is, we can use different aggregations at different levels as well as at different branches of the same level of the hierarchical fuzzy signature structure. We argue that these properties help fuzzy signatures to model problems similarly to the nature of human approaches to problem solving. An important benefit of the fuzzy signature concept is that it can be used to compare the degree of similarity or dissimilarity of two objects with slightly different descriptor structure, which have nevertheless the same main fuzzy signature skeleton. Additionally, fuzzy signatures are capable of dealing with missing input data. Thus, medical and economic diagnoses are obvious application areas for fuzzy signatures.

In [2] we further enhanced the technique of inference in fuzzy signatures, by introducing the Weighted Relevance Aggregation method. The concept behind the Weighted Relevance aggregation method is that the weights in each branch of the fuzzy signature express the perceptions of the relevance of that branch to its higher-level branches in the hierarchical fuzzy signature structure. Thus, this method introduces additional expert knowledge to the fuzzy signature structure to classify vague data. Further, in [2] we proposed to use simple aggregation functions: minimum, average, maximum-average, and maximum with these weights. In [4] we showed a methodology of learning these weights for Weighted Relevance Aggregation, from real world data, using a gradient descent method. In [3] a steepest gradient descent method was successfully used for learning these weights for Weighted Relevance Aggregation in hierarchical fuzzy signatures. But still, we need to manually set the aggregation functions. 
In this paper, we further generalise these weights and aggregation functions into an operator called Weighted Relevance Aggregation Operator (WRAO). This allows us to learn both the aggregation function and the Weighted Relevance at the same time for an individual node in the hierarchical fuzzy signatures structure. Also, we discuss the partial derivatives of the function in question that are needed for gradient based leaning.

In Section 2, we briefly discuss the theory of fuzzy signatures and the Weighted Relevance Aggregation method. In Section 3 we introduce the generalised Weighted Relevance Aggregation Operator and discuss the partial derivatives used. In Section 4 we discuss the methodology of learning this new operator from data, using Guass-Newton optimization method. Finally, in Section 5, we show how to calculate the Jacobian matrix for the Guass-Newton method using a real world application.

\section{Fuzzy Signatures and Weighted Relevance Aggregation}

In this section we discuss the theoretical background of hierarchical fuzzy signature structures and the weighted relevance aggregation method.

Fuzzy signatures are iterative vector valued fuzzy sets, where each vector component can be a further vector valued fuzzy set [8]. A fuzzy signature $s$ can be defined as,

$$
s: X \rightarrow\left[a_{i}\right]_{i=1}^{k}, \text { where } a_{i}=\left\{\begin{array}{c}
{[0,1] ; \text { if leaf }} \\
{\left[a_{i j}\right]_{j=1}^{k_{i}} ; \text { if branch }}
\end{array}\right.
$$

Fig.1 shows an example of a hierarchical fuzzy signature structure with two arbitrary levels $g$ and $(g+1)$. Now, aggregation of an arbitrary branch $a_{q . i}$ in level $g$ (fig. 1 ) can be written as, $a_{q . i}=@_{q . i}\left\{a_{q . i j}\right\}$, where $@_{q . i}$ is an arbitrary aggregation function, $j=1, \ldots n$, and $a_{q . i j} \in[0,1]$. These aggregation functions, which are used for inference in fuzzy signatures, can be simple aggregations like minimum (min), average (avg), maximum_average (max-avg), or maximum (max), which were used in [2], or they can be complex aggregation functions as proposed in [8].

The Weighted Relevance Aggregation concept was proposed in [2] to provide the incorporation of additional expert knowledge to the fuzzy signature structure by introducing the weighted relevance of each branch to its higher-level branches of the fuzzy signature structure. Thus, the Weighted Relevance will give an additional ability to fuzzy signatures for

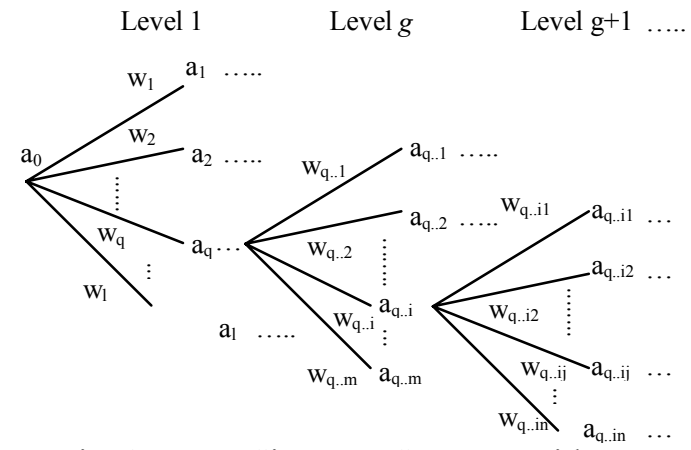

Fig. 1. Fuzzy Signature Structure with two arbitrary levels $g$ and $(\mathrm{g}+1)$

decision making in situations where input data are vague and complex.

The Weighted Relevance aggregation of an arbitrary branch $a_{q \ldots i}$ in a fuzzy signature (Fig.1) can be defined as $a_{q \ldots i}=@_{q \ldots i}\left\{w_{q \ldots i j} \bullet a_{q \ldots i j}\right\}$, where $@_{q . . i}$ is an arbitrary aggregation function, $j=1, \ldots n$, and $a_{q \ldots i j} \in[0,1]$. The following properties hold for arbitrary weighted relevance $w_{q}$ :

i. $w_{q} \in[0,1]$

ii. Note that $\sum_{q=1}^{l} w_{q}$ is not necessarily equal to 1 .

In [1], [2] and [3] we have shown that the Weighted Relevance Aggregation can be used to enhance the accuracy of the final results. Also, it helps to find a more general hierarchical structure, which represents large number of data points for fuzzy signatures.

\section{Weighted Relevance Aggregation Operator (WRAO)}

In this section we first discuss the idea of generalizing weighted relevance and aggregations into an operator called Weighted Relevance Aggregation Operator (WRAO) for fuzzy signatures. Secondly, we obtain the partial derivatives of WRAO for gradientbased learning.

\subsection{Definition and Properties of WRAO}

The importance of WRAO is that it enhances the adaptability of the hierarchical fuzzy signature structure by giving more flexibility to learn better values, which are weighted relevance and aggregation functions, for inference.

The generalised means [5] of $n$ arguments $s_{1}, s_{2}, \ldots, s_{n} \in[0,1]$ is a function $g:[0,1]^{n} \rightarrow[0,1]$ such that, 


$$
g\left(s_{1}, s_{2}, \ldots, s_{n}\right)=\left(\frac{1}{n} \sum_{i=1}^{n} s_{i}^{p}\right)^{\frac{1}{p}}
$$

where $p \in \Re$ and $p \neq 0$. We used the above function to define the generalised WRAO for fuzzy signatures.

Definition: The generalised WRAO of $n$ branches $s_{1}, s_{2}, \ldots, s_{n} \in[0,1]$ with $n$ weighted relevancies $w_{1}, w_{2}, \ldots, w_{n} \in[0,1]$, in a fuzzy signature, is a function $g:[0,1]^{2 n} \rightarrow[0,1]$ such that,

$g\left(s_{1}, s_{2}, \ldots, s_{n} ; w_{1}, w_{2}, \ldots, w_{n}\right)=\left(\frac{1}{n} \sum_{i=1}^{n}\left(w_{i} s_{i}\right)^{p}\right)^{\frac{1}{p}}$

where $p \in \Re \quad, p \neq 0, \quad i \in[1, n] \quad$ and $\quad \sum_{i=1}^{n} w_{i}$ is not necessarily equal to 1 . We called $p$ is the aggregation factor of the above function.

Theorem 1: WRAO (2) holds following properties,

(a) Partially Idempotent w.r.t $\mathrm{s}_{\mathrm{i}}$ when all $\mathrm{w}_{\mathrm{i}}$ are fixed and vice versa.

(b) Commutativity.

(c) Partially Monotonic w.r.t $\mathrm{s}_{\mathrm{i}}$ when all $\mathrm{w}_{\mathrm{i}}$ are fixed and vice versa.

Proof (a): The classical Idempotency does not hold for (2). Instead, it can be proof that (2) is partially idempotent concerning $s_{i}$ if the values of $w_{i}$ are fixed, or concerning $\mathrm{w}_{\mathrm{i}}$ if the values of $\mathrm{s}_{\mathrm{i}}$ are fixed. Let $a^{*}=w_{i} a_{i} \forall i \in[1, n]$, and $a^{*} \geq 0$ in (2). Also, assume that all $\mathrm{w}_{\mathrm{i}}$ are fixed. Then we get from (2),

$$
\left[\frac{\left(a^{* p}+a^{* p}+. .+a^{* p}\right)}{n}\right]^{\frac{1}{p}}=\left(\frac{n a^{* p}}{n}\right)^{\frac{1}{p}}=a^{*} \text { 。 }
$$

Similarly, we can proof that, (2) is partially idempotent concerning $w_{i}$ if the values of $s_{i}$ are fixed.

Proof (b): Commutativity of (2) is obvious。 Proof (c): (2) is Monotonic function, $g\left(s_{1}, \ldots, s_{i}^{\prime}, \ldots, s_{n} ; w_{1}, w_{2}, \ldots, w_{n}\right) \geq g\left(s_{1}, s_{2}, \ldots, s_{n} ; w_{1}, w_{2}, \ldots, w_{n}\right)$, if $s_{i}^{\prime} \geq s_{i}$ for an $i \in[1, n]$. Also, assume that all $\mathrm{w}_{\mathrm{i}}$ are fixed. Now, we can write that,

$$
\left(w_{i} s_{i}^{\prime}\right)^{p} \geq\left(w_{i} s_{i}\right)^{p} \geq 0
$$

as $s_{i}^{\prime} \geq s_{i}$ for $i \in[1, n]$ and by definition, $\left(w_{1} s_{1}\right)^{p}+. .+\left(w_{i} s_{i}\right)^{p}+. .+\left(w_{n} s_{n}\right)^{p} \geq\left(w_{1} s_{1}\right)^{p}+\left(w_{2} s_{2}\right)^{p}+. .+\left(w_{n} s_{n}\right)^{p}$

This follows that,

$$
\begin{aligned}
& \left(\frac{\left(w_{1} s_{1}^{\prime}\right)^{p}+\left(w_{2} s_{2}^{\prime}\right)^{p}+. .+\left(w_{n} s_{n}^{\prime}\right)^{p}}{n}\right)^{\frac{1}{p}} \\
& \geq\left(\frac{\left(w_{1} s_{1}\right)^{p}+\left(w_{2} s_{2}\right)^{p}+. .+\left(w_{n} s_{n}\right)^{p}}{n}\right)^{\frac{1}{p}} \text { 。 }
\end{aligned}
$$

Similarly, we can proof that, (2) is partially monotonic concerning $\mathrm{w}_{\mathrm{i}}$ if the values of $\mathrm{s}_{\mathrm{i}}$ are fixed.

Theorem 2: WRAO (2) shows following characteristics,
(a) $p \rightarrow 0$ then WRAO $\rightarrow$ geometric mean
(b) $\lim _{p \rightarrow+\infty} g\left(s_{1}, s_{2}, \ldots, S_{n} ; w_{1}, w_{2}, \ldots, w_{n}\right)=\max \left(w_{1} S_{1}, w \underline{z}_{2}, \ldots, w_{n} S_{n}\right)$
(c) $\lim _{p \rightarrow-\infty} g\left(s_{1}, s_{2}, \ldots, s_{n} ; p ; w_{1}, w_{2}, \ldots, w_{n}\right)=\min \left(w_{1} s_{1}, w_{2} s_{2}, \ldots, w_{n_{n}}\right)$
(d) $p=1$ then WRAO $\rightarrow$ arithmetic mean
(e) $p \rightarrow-1$ then WRAO $\rightarrow$ harmonic mean

Proof (a): $p \rightarrow 0$

$$
\begin{aligned}
& \left(\frac{1}{n} \sum_{i=1}^{n}\left(w_{i} S_{i}\right)^{p}\right)^{\frac{1}{p}}=\left(\frac{\left(w_{1} S_{1}\right)^{p}+\left(w_{2} s_{2}\right)^{p}+. .+\left(w_{n} S_{n}\right)^{p}}{n}\right)^{\frac{1}{p}} \\
& \lim _{p \rightarrow 0} \ln \left(\frac{\left(w_{1} s_{1}\right)^{p}+\left(w_{2} s_{2}\right)^{p}+. .+\left(w_{n} s_{n}\right)^{p}}{n}\right)^{\frac{1}{p}} \\
& =\lim _{p \rightarrow 0} \frac{\ln \left(\left(w_{1} S_{1}\right)^{p}+\left(w_{2} S_{2}\right)^{p}+. .+\left(w_{n} S_{n}\right)^{p}\right)-\ln (n)}{p} \\
& =\lim _{p \rightarrow 0} \frac{d}{d p}\left[\ln \left(\left(w_{1} S_{1}\right)^{p}+\left(w_{2} s_{2}\right)^{p}+. .+\left(w_{n} s_{n}\right)^{p}\right)\right] \text { L'Hospital rule } \\
& =\lim _{p \rightarrow 0} \frac{\left(w_{1} S_{1}\right)^{p} \ln \left(w_{1} S_{1}\right)+\left(w_{2} S_{2}\right)^{p} \ln \left(w_{2} s_{2}\right)+. .+\left(w_{n} S_{n}\right)^{p} \ln \left(w_{n} S_{n}\right)}{\left(w_{1} S_{1}\right)^{p}+\left(w_{2} S_{2}\right)^{p}+. .+\left(w_{n} S_{n}\right)^{p}} \\
& =\ln \left(w_{1} s_{1} \cdot w_{2} s_{2} \cdots \cdot w_{n} s_{n} \frac{1}{n}\right.
\end{aligned}
$$

As logarithm is strictly monotonic increasing function,

$$
\lim _{p \rightarrow 0}\left(\frac{1}{n} \sum_{i=1}^{n}\left(w_{i} s_{i}\right)^{p}\right)^{\frac{1}{p}}=\left(w_{1} S_{1} \cdot w_{2} s_{2} \cdot \ldots \cdot w_{n} s_{n}\right)^{\frac{1}{n}} \text { 。 }
$$

Proof (b): $p \rightarrow+\infty$; let $\max _{1}^{n}\left(w_{i} s_{i}\right)=a^{*}$ and divide the denominator and numerator of (2) by $a^{* p}$ (suppose that, always $\exists i \in[1, n]$ s.t. $a^{*} \neq 0$; otherwise there is no meaning of the aggregation).

$$
\begin{aligned}
& \lim _{p \rightarrow \infty}\left(\frac{1}{n} \sum_{i=1}^{n}\left(w_{i} s_{i}\right)^{p}\right)^{\frac{1}{p}}=\lim _{p \rightarrow \infty}\left(\frac{\frac{\left(w_{1} s_{1}\right)^{p}}{a^{* p}}+\frac{\left(w_{2} s_{2}\right)^{p}}{a^{* p}}+. .+\frac{\left(w_{n} s_{n}\right)^{p}}{a^{* p}}}{\frac{n}{a^{* p}}}\right)^{\frac{1}{p}} \\
& \text { if }\left(\frac{w_{n} s_{n}}{a^{*}}\right)<1 \text { then }\left(\frac{w_{n} s_{n}}{a^{*}}\right)^{p}=0 ; \text { and } \\
& \text { if }\left(w_{n} s_{n}\right)=a^{*} \text { then } \lim _{p \rightarrow \infty}\left(\frac{w_{n} s_{n}}{a^{*}}\right)^{p}=1 \\
& =\lim _{p \rightarrow \infty}\left(\frac{k}{n / a^{* p}}\right)^{\frac{1}{p}} \text { where } k=\sum_{i=1 \mid\left(w_{i} s_{i}\right)=a^{*}}^{n} 1 \text { and } k \leq n \\
& =\lim _{p \rightarrow \infty}\left(\frac{k}{n}\right)^{\frac{1}{p}} a^{*}=a^{*}=\max \left(w_{1} s_{1}+w_{2} s_{2}+. .+w_{n} s_{n}\right) .
\end{aligned}
$$

Proof (c): $p \rightarrow-\infty$; let $\min _{1}^{n}\left(w_{i} s_{i}\right)=a^{*}$ and divide the denominator and numerator of (2) by $a^{* p}$. 


$$
\begin{aligned}
& \lim _{p \rightarrow-\infty}\left(\frac{1}{n} \sum_{i=1}^{n}\left(w_{i} s_{i}\right)^{p}\right)^{\frac{1}{p}}=\lim _{p \rightarrow-\infty}\left(\frac{\frac{\left(w_{1} s_{1}\right)^{p}}{a^{* p}}+\frac{\left(w_{2} s_{2}\right)^{p}}{a^{* p}} . .+\frac{\left(w_{n} s_{n}\right)^{p}}{a^{* p}}}{\frac{n}{a^{* p}}}\right)^{\frac{1}{p}} \\
& \text { if }\left(\frac{w_{n} s_{n}}{a^{*}}\right)>1 \text { then } \lim _{p \rightarrow-\infty}\left(\frac{w_{n} s_{n}}{a^{*}}\right)^{p}=0 \text {; and } \\
& \text { if }\left(w_{n} s_{n}\right)=a^{*} \text { then } \lim _{p \rightarrow-\infty}\left(\frac{w_{n} s_{n}}{a^{*}}\right)^{p}=1 \\
& =\lim _{p \rightarrow-\infty}\left(\frac{k}{n / a^{* p}}\right)^{\frac{1}{p}} \text { where } k=\sum_{i=1 \mid\left(w_{i} s_{i}\right)=a^{*}}^{n} 1 \text { and } k \leq n \\
& =\lim _{p \rightarrow-\infty}\left(\frac{k}{n}\right)^{\frac{1}{p}} a^{*}=a^{*}=\min \left(w_{1} s_{1}+w_{2} s_{2}+. .+w_{n} s_{n}\right) \text { 。 }
\end{aligned}
$$

Proof: (c) $p \rightarrow 1$; proof is obvious, apply $\mathrm{p}=1$, $\left(\frac{1}{n} \sum_{i=1}^{n}\left(w_{i} s_{i}\right)\right)=\left(\frac{\left(w_{1} s_{1}\right)+\left(w_{2} s_{2}\right)+. .+\left(w_{n} s_{n}\right)}{n}\right)$ 。

Proof: (d) $p \rightarrow-1$; proof is obvious, apply $\mathrm{p}=-1$, $\left(\frac{1}{n} \sum_{i=1}^{n}\left(w_{i} s_{i}\right)^{-1}\right)^{-1}=\left(\frac{n}{\frac{1}{w_{1} s_{1}}+\frac{1}{w_{2} s_{2}}+. .+\frac{1}{w_{n} s_{n}}}\right)$.

Now, it is clear that our WRAO generates a class of aggregations, which span the universe of all aggregations which fall between Zadeh's classical conjunction and disjunctions operators, also including these two operators. Thus, we argue that WRAO enhances the adaptability of hierarchical fuzzy signature structures to different applications. In addition, it simplifies the learning of fuzzy signature models from data, by generalizing both the Weighted Relevance and aggregation functions into one operator.

\subsection{Partial Derivatives of WRAO}

The Weighted Relevance aggregation of an arbitrary branch $a_{q \ldots i}$ in a fuzzy signature (Fig.1), using WRAO, can be written as,

$a_{q \ldots i}=\left[\frac{1}{n} \sum_{j=1}^{n}\left(w_{q \ldots i j} a_{q \ldots i j}\right)^{p_{q \ldots i}}\right]^{\frac{1}{p_{q \ldots i}}}$

where $n$ is the number of leaves in the branch $q \ldots i$, $w_{q \ldots i j}$ is the Weighted Relevance of the leaf $a_{q \ldots i j}$, where $j \in[1, n]$, and $p_{q \ldots i}$ is the aggregation factor of branch $q \ldots i$.

To avoid the constraints on the weighted relevance factor $w_{i}$, we replaced it by the following sigmoid function [3], [4]:

$$
w_{i}=\frac{1}{1+e^{-\lambda_{i}}} \text {, }
$$

where $\lambda_{i} \in \Re$. After the above transformation it becomes clear that for any values of the parameter $\lambda_{i}$ the weighted relevancies are $w_{i} \in[0,1]$ and $\sum w_{i}$ is not necessarily equal to 1 . Therefore the constrained optimization problem has been transformed into an unconstrained optimization problem. Now equation (4) can be rewritten as follows,

$a_{q \ldots i}=\left[\frac{1}{n} \sum_{j=1}^{n}\left(\left\{\frac{1}{1+e^{-\lambda_{q \ldots i j}}}\right\} a_{q \ldots i j}\right)^{p_{q \ldots i}}\right]^{\frac{1}{p_{q \ldots i}}}$

This form of WRAO equation can be used for gradient based learning. The parameters we need to learn are the aggregation factor $p_{q \ldots i}$, and the Weighted Relevance factor $\lambda_{q . . i}$. First, we can obtain the partial derivatives of the equation (5) w.r.t. $p_{q \ldots i}$.

$\frac{\partial a_{q \ldots i}}{\partial p_{q \ldots i}}=\left[\frac{a_{q \ldots i}^{\left(1-p_{q . . i}\right)}}{n \cdot p_{q_{\ldots i}}^{2}}\right]\left\{\sum_{j=1}^{n} t \ln (t)-n a_{q \ldots i}^{p_{q . i i}} \ln \left(a_{q \ldots i}^{p_{\ldots . i .}}\right)\right\}$

where $t=\left(a_{q_{\ldots i j}}\left[w_{q \ldots i j}\right]\right)^{p_{q \ldots i}}$. Similarly, we can obtain the partial derivatives of the equation (5) w.r.t. $\lambda_{q . . i k}$.

$\frac{\partial a_{q . i i}}{\partial \lambda_{q . i k}}=\left(\frac{1}{p_{q . i i}}\right)\left[\frac{1}{n} \sum_{j=1}^{n}\left(w_{q . . i j} a_{q . . i j}\right)_{p_{q . i .}}\right]^{\left(\frac{1}{p_{q . i .}}-1\right)} \frac{d\left(\left[\frac{w_{q . i k} a_{q . i k}}{n}\right]^{p_{q . i i}}\right)}{d \lambda_{q . i k}}$ where $k \in[1, n]$.

\section{Gauss-Newton Optimization method for WRAO Learning}

This section reviews the Newtons' gradient based optimization method for learning. The Newtons' method is an advanced optimization algorithm that outperforms simple gradient descent when applied in a wide variety of problems.

Newtons' method uses quadratic approximation to the objective function that matches first and second derivative values at that point. Newton's method is a second order derivative method [10].

The Sum of Square Errors (SSE) can be use as the error evaluation criteria,

$$
f(s)=\frac{1}{2} \sum_{i=1}^{n}\left(t_{i}-s_{i}\right)^{2}=\frac{1}{2}\|\underline{t}-\underline{s}\|^{2}=\frac{1}{2}\|\underline{e}\|^{2}
$$

where $t$ stands for the target vector, $\underline{s}$ for the actual output vector of the fuzzy signature, and \|\| denotes the 2-norm. Also, it will be assumed that there are $m$ parameters to be learnt and there are $n$ records in the training data set, such that $n>m$.

The next iteration, $k+1$, of the of the Newtons' method can be calculated as,
(a) $G^{k} \cdot \underline{u}^{k}=-g^{k}=-J^{k} \cdot \underline{e}^{k}$ 
(b) $\operatorname{par}^{k+1}=\underline{p a r}^{k}+\underline{u}^{k}$

where $\mathrm{g}^{\mathrm{k}}$ is the gradient vector, $\mathrm{G}^{\mathrm{k}}$ is the Hessian, and $J$ is the Jacobian matrix of (8), $\underline{u}^{k}$ is the next update vector, and the vector $\underline{p^{k} r^{k}}$ contains the all parameters needing to be optimised by the learning algorithm in the $k^{\text {th }}$ iteration. The Jacobian matrix of (8) can be written as

$J=\left[\frac{\partial\left(\underline{e}^{k}\right)}{\left.\partial \underline{p^{k a r}}\right)^{k}}\right]$

where $k$ is the iteration number of the training algorithm.

\section{Example Problem: Salary Selection Fuzzy Signature}

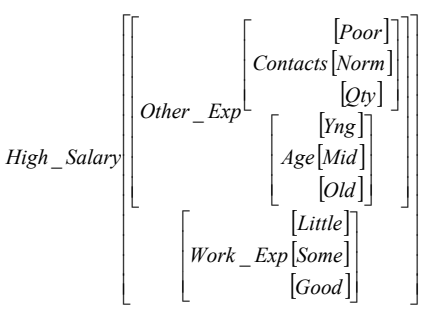

Fig.2. High Salary Selection fuzzy signature

In this section we describe how to calculate the Jacobian matrix for gradient based learning, discussed in Section 4, using the partial derivatives shown in Section 3. The High Salary Selection fuzzy signature (Fig.2) in [2] has been selected as the example real

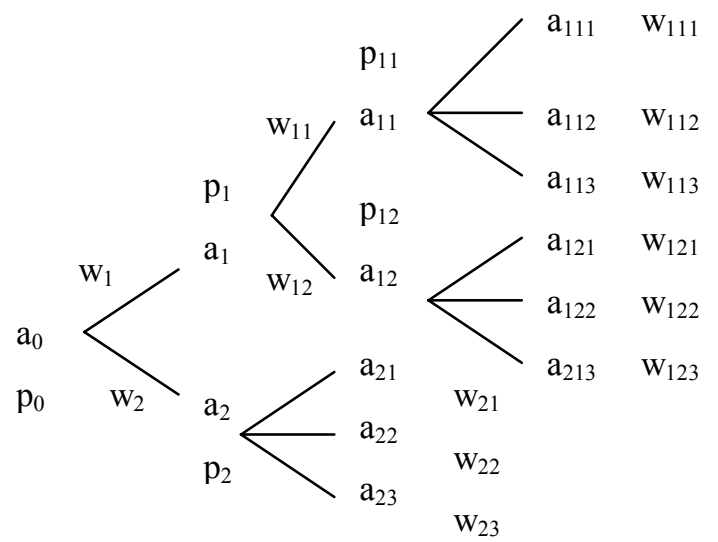

Fig.3 High Salary Selection fuzzy signature with input values

world problem. The High Salary Selection fuzzy signature in Fig. 2 describes the prediction of high salary of a person given by work experience, contacts and age.
Fig. 3 shows the same High Salary Selection fuzzy signature structure with input values and the parameters, which are to be learnt, at each node. The parameters $w_{i}$ and $p_{i}$ represent the Weighted Relevance and aggregation factor at node $i$ respectively.

Now, let us assume that we need to calculate the Jacobian matrix for the above fuzzy signature for the $k^{\text {th }}$ iteration of the learning. The Jacobian matrix for the High Salary Selection fuzzy signature is of the following form:

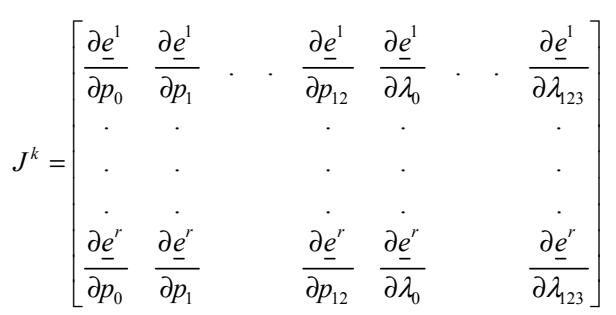

where $r$ is the number of records in the training data set. The Chain Rule can be used to find the partial derivatives of the above Jacobian matrix.

\section{Conclusion}

We proposed WRAO for inference in hierarchical fuzzy signature structures. WRAO generalised both the Weighted Relevance and the aggregation functions in hierarchical fuzzy signatures, into a single operator. The requirements and benefits of the WRAO have been pointed out. Secondly, we have discussed the learning WRAO's from real world data. Also, the partial derivatives of WRAO have been obtained for gradient-based learning. Finally, a practical example has been used to show the technique of calculating the Jacobian matrix for the gradient based learning.

\section{References}

[1] B.S.U. Mendis, T.D. Gedeon, L.T. Kóczy, "Flexibility and Robustness of Hierarchical Fuzzy Signature Structures with Perturbed Input Data," International Conf. of Information Processing and Management of Uncertainty in Knowledge Based Systems (IPMU), Paris, 2006.

[2] B.S.U. Mendis, T.D. Gedeon, L.T. Kóczy, "Investigation of Aggregation in Fuzzy Signatures", 3rd International Conference on Computational Intelligence, Robotics and Autonomous Systems, Singapore, December 2005.

[3] B.S.U. Mendis, T.D. Gedeon, L.T. Kóczy, "Learning Weights from Observations for Hierarchical Fuzzy Signatures ," SCIS/ISIS, Japan, 2006 (accepted). 
[4] B.S.U. Mendis, T.D. Gedeon, L.T. Kóczy, "On the Issue of Learning Weights from Observations for Fuzzy Signatures," World Automation Congress (WAC), Hungary, 2006.

[6] H. Dyckhoff, W. Pedrycz, Generalized means as model of compensative connectivities, Fuzzy Sets and Systems 14, 143-154, 1984.

[7] L.T. Kóczy, "Vector Valued Fuzzy Set," Busefal, ete 1980, pp. 41-57.

[8] L.T. Kóczy, T. Vámos, G. Biró, "Fuzzy Signatures," Proc. of EUROFUSE-SIC '99, 1999, pp. 210-217.

[9] T. Vámos, L.T. Kóczy, G. Biró, "Fuzzy Signatures in Data Mining," Proc. of the joint $9^{\text {th }}$ IFSA World Congress, 2001, pp. 2842-2846.

[10] R. Fletcher, "Practical Methods of Optimization," vol. 1. John Wiley and Sons, 1980. 